\title{
Annihilation characteristics of positrons in free-standing thin metal and polymer films
}

A. Uedono ${ }^{a, *}$, K. Ito ${ }^{a}$, H. Nakamori ${ }^{a}$, S. Ata ${ }^{b}$, T. Ougizawa ${ }^{\text {b }}$, K. Ito ${ }^{\text {c }}$, Y.

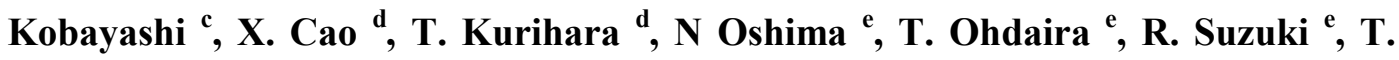

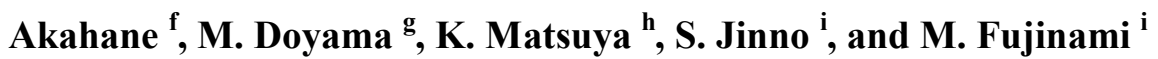

${ }^{a}$ Institute of Applied Physics, University of Tsukuba, Tsukuba, Ibaraki, 305-8573, Japan

${ }^{b}$ Department of Organic and Polymeric Materials, Tokyo Institute of Technology, Meguro-ku, Tokyo 152-8552, Japan

${ }^{c}$ National Metrology Institute of Japan, National Institute of Advanced Industrial Science and Technology, Tsukuba, Ibaraki, 305-8565, Japan

${ }^{d}$ Institute of Materials Structure Science, High Energy Accelerator Research Organization, Tsukuba, Ibaraki, 305-0801, Japan

${ }^{e}$ Research Institute of Instrumentation Frontier, National Institute of Advanced Industrial Science and Technology, Tsukuba, Ibaraki, 305-8568, Japan

${ }^{f}$ Advanced Nanomaterials Lab., National Institute for Materials Science, 1-1 Namiki, Tsukuba, Ibaraki 305-0044, Japan

${ }^{g}$ Teikyo University of Science and Technology, Uenohara, Yamanashi, 409-0193, Japan

${ }^{h}$ Japan Electron Optics Laboratory (JEOL) Ltd., Akishima, Tokyo 196-0021, Japan

${ }^{i}$ Department of Applied Chemistry, Chiba University, Yayoi, Inage, Chiba 263-8522, Japan

Annihilation characteristics of positrons and positronium (Ps) in thin metal and 
polymer films were studied. Monoenergetic positrons were implanted into free-standing thin $\mathrm{W}$ and $\mathrm{Au}$ films, and the annihilation $\gamma$-rays of positron-electron pairs were measured as a function of the incident energy of positrons. At the front-side surfaces of the films, an emission of Ps into vacuum and a resultant self-annihilation of ortho-Ps (o-Ps) were observed. At the backside surfaces, the Ps emission was found to be enhanced by an increase in the numbers of epithermal positrons and/or secondary electrons introduced by the impact of energetic positrons. For thin polymer films (polyester and polyethylene), the emission rate of $o$-Ps from the backside surfaces was higher than that from the metal films, which was attributed to the out-diffusion of $o$-Ps formed in the films. Those results suggested that the emission rate of Ps into vacuum was sensitive to the Ps formation process in the bulk and at the surface.

PACS: 41.75.Fr, 87.50.Gi, and 78.70.Bj

Keywords: positronium; positron; thin film; metal; polymer

*Corresponding author: uedono@sakura.cc.tsukuba.ac.jp 


\section{Introduction}

Positron annihilation is an established technique for characterizing materials $[1,2]$. When a positron is implanted into condensed matter, it annihilates with an electron, resulting in the generation of two $\gamma$-rays of about $511 \mathrm{keV}$. One can determine the momentum distribution of electrons annihilating with positrons from measurements of the Doppler shift in the energy of the $\gamma$-rays. If slow/monoenergetic positron beams are used, the implantation profile of the positrons can be adjusted to the region of interest in the sample by accelerating the positrons to the desired energy [3]. This technique enables us to characterize surfaces and subsurface regions of materials [4]. When a positron penetrates into metals, it quickly loses its energy through plasmon and phonon scattering. Some positrons may diffuse back to the surface, and may be emitted into the vacuum if the positron work function $\left(\phi_{+}\right)$is negative. Alternatively, they may capture an electron and be emitted as positronium (Ps: a hydrogen-like bound state between a positron and an electron) with an energy of a few eV (1-3 eV) depending on the Ps work function and the electronic density of states near the surface. The formation and emission processes of Ps have been studied extensively, and the results show that they provide unique information about surface properties [5-9].

When monoenergetic positrons are implanted into thin films, positrons may interact with electrons at the backside surfaces of the films. The annihilation characteristics of positrons at the backside surfaces may not be the same as those at the front side surface, because of the increase in the secondary electrons generated by energetic positrons and epithermal positrons. A transmission-type positron moderator (thin metal film) is often used to obtain brightness enhanced positron beams $[3,6]$, where the annihilation characteristic of positrons transmitted through thin films affects the moderation 
efficiency of slow positrons. When the positron beam is used as a transmission positron microscope (TPM) [10], an analysis of TPM images will require the detailed knowledge about nature of transmitted positrons. Therefore, a study of positrons at the backside surface is important to enhance their potential. Here, we describe annihilation characteristics of positrons in free-standing metal and polymer thin films using the monoenergetic positron beam technique.

The energy of $2 \gamma$ annihilation photons of positron-electron pairs is given by the relation $E_{\gamma}=511 \pm \Delta E_{\gamma} \mathrm{keV}$. The Doppler shift, $\Delta E_{\gamma}$, is given by $\Delta E_{\gamma}=p_{\mathrm{L}} c / 2$, where $c$ is the speed of light and $p_{\mathrm{L}}$ is parallel to the direction of the $\gamma$ rays. In metals, a freely diffusing positron may annihilate with electrons from the delocalized state, or it may be trapped by vacancy-type defects before the annihilation. Thus, one can identify the annihilation mode of positrons by measuring the Doppler broadening spectra of the annihilation radiation. The resultant changes in the spectra are characterized by the $S$ parameter, which mainly reflects the changes due to the annihilation of positron-electron pairs with a low-momentum distribution. The annihilation characteristics of positrons in polymers are different from those described above, and details are described elsewhere [2].

Ps exhibits two spin states: para-Ps ( $p$-Ps) is a singlet state (spin antiparallel bound state between a positron and an electron) and ortho-Ps (o-Ps) is a triplet state (spin parallel bound state). The ratio of the formation probabilities of $p$-Ps to $o$-Ps is $1: 3$. Because the $p$-Ps state decays into two photons with the energy of each photon being close to $511 \mathrm{keV}$, the $S$ value is sensitive to the annihilation probability of $p$-Ps $[11,12]$. In vacuum, $o$-Ps primarily exhibits three-photon $(3 \gamma)$ annihilation that produces a continuous energy distribution for each of the quanta ranging from approximately 0 to 
$511 \mathrm{keV}$. This annihilation mode can be characterized by $V / T$, where $T$ denotes the counts corresponding to both the three- and two-photon processes and $V$ denotes the counts corresponding to the three-photon process.

\section{Experiment}

The samples used in the present experiments were free-standing metal and polymer thin films. The $0.2-\mu \mathrm{m}$-thick tungsten (W) and $0.5-\mu \mathrm{m}$-thick gold $(\mathrm{Au})$ films were provided by Aarhus University and Goodfellow Co., respectively. The 0.9- $\mu$ m-thick polyester film was supplied by Mitsubishi Polyester Film Co. The 1- $\mu$ m-thick polystyrene film was prepared by the spin-coating method. Details of the preparation of thin polymer films are described elsewhere [13]. Ethylene-vinylalcohol/water solution was spin-coated onto $\mathrm{Si}$ substrates, and then polystyrene/toluene solution $\left(M_{\mathrm{w}}=420\right.$ $\mathrm{kg} / \mathrm{mol}, \mathrm{PDI}=3.3)$ was deposited onto the substrate. After annealing at $120^{\circ} \mathrm{C}(12 \mathrm{~h})$, the polystyrene film was removed from the substrate by dissolving ethylene-vinylalcohol into water. In the present experiments, no surface treatment was done.

The experimental arrangement used in the present study is shown in Fig. 1. Monoenergetic positrons were implanted into the films, and the energy distribution of the annihilation radiation was measured by a hyper-pure Ge detector as a function of the incident energy of positrons $E$. The energy of positrons was varied from $0.1 \mathrm{keV}$ to 29 $\mathrm{keV}$. The mean implantation depth of positrons $\bar{z}$ can be obtained by the relation, $\bar{z}=A E^{n} / \rho . A$ and $n$ are empirical parameters $\left(2.75 \mu \mathrm{g} \mathrm{cm}^{-2} \mathrm{keV}^{-\mathrm{n}}\right.$ and 1.7$)$, and $\rho$ is the mass density. The implantation profile of positrons $P(z)$ can be represented using Makhovian distribution: $\quad P(z)=\left(m z^{m-1} / z_{0}^{m}\right) \exp \left[-\left(z / z_{0}\right)^{m}\right]$, where $m=2$ and 
$z_{0}=\bar{z} / \Gamma(1+1 / m)[1]$. Using those equations, for example, the mean positron energy corresponding to the thickness of the $\mathrm{W}$ film can be calculated to be $18 \mathrm{keV}$, and at this energy, about half number of positrons transmits through the film.

The energy resolution of the Ge detector was about $1.3 \mathrm{keV}$ (full-width-at-half-maximum, FWHM). The angular acceptance of the detector covers the major annihilation events of positrons in the film and those of Ps emitted from the surfaces. Transmitted positrons were guided into a beam dump. The signal-to-noise ratio for the measurements of Doppler broadening spectra was $1.2 \times 10^{3}$, and the effect that the positrons annihilated in the beam dump had on the value of $S$ was almost negligible. The low-momentum part of the Doppler broadening spectrum was characterized by the $S$ parameter, defined as the number of annihilation events over the energy range of $511 \mathrm{keV} \pm \Delta E_{\gamma}$ (where $\Delta E_{\gamma}=0.76 \mathrm{keV}$ ) around the center of the peak. The high-momentum part was characterized by the $W$ parameter, which was calculated from the tail of the peak in the range of $3.4 \mathrm{keV} \leq\left|E_{\gamma}-511 \mathrm{keV}\right| \leq 6.7 \mathrm{keV}$. The relationship between $S$ and $E$ for the $\mathrm{W}$ and Au films was analyzed by VEPFIT, a computer program developed by van Veen et al. [14]. Details of the fitting model and its procedure are described elsewhere $[14,15]$. The self-annihilation of $o$-Ps $(3 \gamma \mathrm{s})$ was characterized by $V / T$, where the energy windows corresponding to $V$ and $T$ were defined as $110-480$ and $110-620 \mathrm{keV}$, respectively.

\section{Results and Discussion}

Figure 2 shows the $S$ values for the $\mathrm{Au}, \mathrm{W}$, polyester, and polystyrene films as functions of $E$. The statistical error of the $S$ value was close to the size of symbols used 
in the figure. For the metal films, the $S$ value was almost constant at $E=8-9 \mathrm{keV}$. This indicates that in this energy range, almost all positrons annihilate in the film without diffusing back to the subsurface region. Large $\mathrm{S}$ values at low $E(\cong 0.1 \mathrm{keV})$ are due to the annihilation of positrons and Ps at the front-side surfaces $[3,4]$. We note that the $S$ values at $E<7 \mathrm{keV}$ were larger than the value of $S_{\text {film. }}$. Since a certain amount of positrons implanted within this energy range can diffuse back to the front-side surface, the $S$ value at $E<7 \mathrm{keV}$ was observed as a weighted average of the $S$ value at the front-side surface $\left(S_{\mathrm{FS}}\right)$ and $S_{\text {Film. }}$. The diffusion length of positrons toward the surface $\left(L_{\mathrm{d}}\right)$ was obtained through fitting of the observed $S-E$ curve at $E=0-10 \mathrm{keV}$. The solid curves for the metal films show the fitting results. The derived values of $L_{\mathrm{d}}$ were $9.5 \pm$ $0.3 \mathrm{~nm}$ for $\mathrm{Au}$ and $15 \pm 1 \mathrm{~nm}$ for $\mathrm{W}$. These were shorter than typical values (typically 150-200 nm) for defect-free metals $[16,17]$. The observed short diffusion lengths were due to the trapping or scattering of positrons by defects in the films. In the fitting procedure, an effect of epithermal positrons [6] on the $S$ values was included in order to reproduce the $S-E$ curves in a low energy region $(E=0-1 \mathrm{keV})$.

The $S$ value started to increase above $10 \mathrm{keV}$, which can be attributed to the annihilation of positrons and Ps at the backside surfaces of the films. For both films, although the values of $S_{\mathrm{FS}}$ differed from each other, the $S$ values seemed to saturate at a constant $S$ value (0.46) at high $E(25-30 \mathrm{keV})$. In this energy region, about $70 \%$ of the positrons were transmitted through the films, and they were guided into the beam dump. When all positrons annihilated in the beam dump, no significant signal was observed around $511 \mathrm{keV}$. This means that the annihilation radiation of transmitted positrons did not contribute to the observed Doppler broadening spectra. With the energy widths used for the calculation of the $S$ value, the calculated $S$ value corresponding to this 
background spectrum was 0.35 . In Fig. 2 , at $E=10-30 \mathrm{keV}$, since the $S$ values increased with increasing $E$, the saturation of the $S$ value at the high energy region was not due to the effect of the background.

For the polymer films, the values of $S_{\text {Film }}(E \cong 5 \mathrm{keV})$ were larger than those for the metal films. In those samples, some positrons annihilate from the Ps states [2]. Because the $S$ value corresponding to the self-annihilation of $p$-Ps is large, the characteristic $S$ values for the polymer films are larger than those for the metal films. The value of $S_{\mathrm{FS}}$

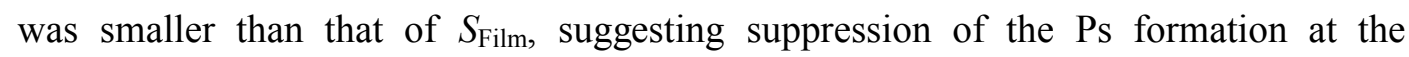
front-side surface. In amorphous polymers, Ps is formed mainly by the reaction between a positron and an excess electron in the spur, where the spur is the terminal track of positrons $[2,18]$. The formation probability of Ps at the front-side surface is thought to be lower than that at the back-side for several reasons, such as an out-diffusion of positrons and spur electrons into the vacuum and/or the decrease in the number of spur electrons due to the low incident energy of positrons [19].

For the polymer films, the $S$ value started to decrease above $10 \mathrm{keV}$ (which can be attributed to the same origin discussed above), but it seemed to approach the characteristic $S$ value for the backside surface $\left(S_{\mathrm{BS}}\right)$ of the metal films. Figure 3 shows the $S-W$ trajectories for the $\mathrm{W}$ and polystyrene films. The details of this graphical analysis are described elsewhere [1]. Arrows in the figure indicate behaviors of the $(S, W)$ values with increasing incident energy of positrons. The open circles show the $(S, W)$ values corresponding to the positron annihilation in the films. For the $\mathrm{W}$ films, the $(S, W)$ values from the front-side surface and to the film can be represented by a straight line, and those from the film to the backside surface can be represented by another straight line. This suggests that the number of major annihilation modes was 
three, and the annihilation characteristics of positrons at the front-side surface were different from those at the backside surface. For the polystyrene film, a similar behavior of $(S, W)$ was obtained, and the $(S, W)$ value at the backside surfaces was close to that for the $\mathrm{W}$ film. For the $\mathrm{Au}$ and polyester films, the $(S, W)$ values at the backside surface were close to those for the $\mathrm{W}$ and polystyrene films (not shown in Fig. 3). The Doppler broadening spectra at $E=25-30 \mathrm{keV}$ were successfully fitted using a single Gaussian function, and the derived standard deviation was 2.4-2.5 keV. The annihilation characteristics of positrons near the backside surfaces is therefore unlikely to depend on the materials (metals or polymers), but it could be close to the annihilation of positrons in high-density electron gas formed by secondary electrons. The annihilation of energetic $p$-Ps is also a candidate to explain the broadening of the spectrum.

Figure 4 shows the $V / T-E$ curves for the metal and polymer films. Since Ps is not formed in metals [4], the $V / T$ values for the annihilation of positrons in the metal films $(E=1-10 \mathrm{keV})$ represent values corresponding to positron annihilation without the emission of $3 \gamma \mathrm{s}$. For the metal films, although an increase in the $S$ value was observed with decreasing $E$ in the region between $0 \mathrm{keV}$ and $8 \mathrm{keV}$ (Fig. 2), an increase in the $V / T$ value was observed only at $E=0-1 \mathrm{keV}$; where the effect of epithermal positrons on the $S$ value was dominant. The increase in the $S$ value at $E=1-8 \mathrm{keV}$ is mainly due to the increased number of thermalized positrons able to reach the surface. Thus, the emission rate of Ps formed from thermalized positrons is likely to be low in the present experiments, which can be mainly attributed to the surface contamination of the films. In turn, both the values of $S$ and $V / T$ increased above $10 \mathrm{keV}$. At the backside surface, the number of secondary electrons introduced by the impact of energetic positrons is larger than those at the front-side surface. We attribute the observed high Ps emission 
rate at the backside surface to the high formation probability of energetic Ps formed from energetic positrons and/or secondary electrons.

For the polymer films, the $V / T$ values for the films coincided with those for the metal films. In polymers, the positron involved in $o$-Ps annihilates with one of the surrounding electrons in free volumes [2]. Thus, we concluded that almost all Ps annihilates into $2 \gamma \mathrm{s}$ from this "pick-off process" in the film. Above $E=10 \mathrm{keV}$, the $V / T$ value started to increase, and the observed $V / T$ values in this region were larger than those for the metal films. From measurements of the time-of-flight of Ps emitted from $\mathrm{SiO}_{2}$, Nagashima et al. [9] observed the emission of Ps formed in the bulk. Thus, in the present experiments, a similar out-diffusion of $o$-Ps and subsequent $o$-Ps emission from the backside surface could occur. This additional contribution of Ps could be an origin of the observed large $V / T$ value for the polymer films.

\section{Summary}

We studied annihilation characteristics of positrons and Ps in thin metal and polymer films. At the front-side surfaces of the metal films, the behaviors of the $S$ and $V / T$ values were different, and this discrepancy was attributed to the difference in the emission rates of Ps formed by epithermal and thermalized positrons. At the backside surface, both the $S$ and $V / T$ values increased, suggesting enhanced Ps emission into the vacuum. We attributed this to the formation of energetic Ps by an increased number of epithermal positrons and/or secondary electrons introduced by the impact of positrons. At the front-side surface of the polymer films, the $S$ value was smaller than that for the bulk, which is due to the reduction of Ps formation probability. The decreased numbers of spur electrons at the front side can be a factor that suppresses Ps formation. In turn, 
the $V / T$ value at the backside surface was higher than that in the film. We attributed this to the out-diffusion of $o$-Ps formed in the films. We have shown that the emission rate of Ps into vacuum is sensitive to the Ps formation process in the bulk and at the surface. The observed phenomena in the present experiments could be used to improve a method to generate a Ps beam, which might be useful to study the reaction of Ps.

\section{Acknowledgements}

Part of this study was financially supported by Development of system and technology for advanced measurement and analysis in Japan Science and Technology Agency (JST). 
1. R. Krause-Rehberg and H.S. Leipner, Positron Annihilation in Semiconductors, Solid-State Sciences 127 (Springer-Verlag, Berlin, 1999).

2. Principles and Applications of Positron and Positronium Chemistry, ed. Y. C. Jean, P. E. Mellon, D. M. Schrader, (World Scientific, Singapore 2003).

3. Positron Beams and Their Applications, ed. P. Coleman (World Scientific, Singapore 2000).

4. P. J. Schultz and K. G. Lynn, Rev. Mod. Phys. 60 (1988) 701.

5. S. Chu, A. P. Mills, Jr., and C. A. Murray, Phys. Rev. B 25 (1981) 2060.

6. A. P. Mills, jr., Positron Solid-State Physics, Proc. of Int. School of Phys. "Enrico Fermi” (North-Holland, Amsterdam, 1983) p. 432.

7. R. H. Howell, I. J. Rosenberg, M. J. Fluss, R. E. Goldberg, and R. B. Laughlin, Phys. Rev. B 35 (1987) 5303.

8. Yen-C. Chen, K. G. Lynn, and B. Nielsen, Phys. Rev. B 37 (1988) 3105.

9. Y. Nagashima, M. Kakimoto, T. Hyodo, K. Fujiwara, A. Ichimura, T. Chang, J. Deng, T. Akahane, T. Chiba, K. Suzuki, B. T. A. McKee, and A. T. Stewart, Phys. Rev. A 52 (1988) 258.

10. M. Doyama, Y. Kogura, M. Inoue, T. Kurihara, T. Yoshiie, and M. Matsuya, Appl. Surf. Sci. 252 , 3126 (2006).

11. A. Uedono, R. Suzuki, T. Ohdaira, T. Uozumi, M. Ban, M. Kyoto, S. Tanigawa, and T. Mikado, J. Polym. Sci. B 36 (1998) 2597.

12. A. Uedono, R. Suzuki, T. Ohdaira, T. Mikado, S. Tanigawa, M. Ban, M. Kyoto, and T. Uozumi, J. Polym. Sci. B 38 (2000) 101.

13. Y. Huang and D. R. Paul, J. Membrane Sci. 244 (2004) 167. 
14. A. van Veen, H. Schut, J. de Vries, R. A. Hakvoort, and M. R. Ijpma: AIP Conf. Proc. 218 (1990) 171.

15. A. Uedono, T. Mori, K. Morisawa, K. Murakami, T. Ohdaira, R. Suzuki, T. Mikado, K. Ishioka, M. Kitajima, S. Hishita, H. Haneda, and I. Sakaguchi, J. Appl. Phys. 93 (2003) 3228 .

16. A. Uedono, S. Tanigawa, and H. Sakairi, J. Nucl. Materials 184 (1991) 191.

17. A. Uedono, T. Suzuki, T. Nakamura, T. Ohdaira, and R. Suzuki, J. Appl. Phys. 98 (2005) 043504.

18. O. E. Mogensen, J. Chem. Phys. 60 (1974) 998.

19. Y. Kobayashi, I. Kojima, S. Hishita, T. Suzuki, E. Asari, and M. Kitajima, Phys. Rev. B 52 (1995) 823. 
Fig. 1. Experimental arrangement for the present experiments. A hyper-pure Ge detector $(\phi 54 \times 53 \mathrm{~mm})$ was used to measure the annihilation radiation of positron-electron pairs. Positrons transmitted through the film were guided into a beam dump.

Fig. 2. $S$ as a function of incident positron energy $E$ for tungsten (W), gold (Au), polyester, and polystyrene films. The statistical error was close to the size of symbols used in the figure. The annihilation of positron-electron pairs near the backside surface was observed to affect the $S$ value at $E>10 \mathrm{keV}$. For the metal films, the solid curves are results of fitting to the experimental data.

Fig. 3. $S-W$ trajectories for the $\mathrm{W}$ and polystyrene films. Arrows in the figure indicate behaviors of the $(S, W)$ values with increasing incident energy of positrons. The open circles represent the $(S, W)$ values corresponding to the positron annihilation in the films. The $(S, W)$ values for the backside surfaces of both films were close to each other.

Fig. 4. $V / T-E$ curves for the $\mathrm{Au}, \mathrm{W}$, polyester, and polystyrene films. The increase in the $V / T$ value corresponds to the increase in the self-annihilation rate of $o$-Ps in vacuum. 
Figure 1

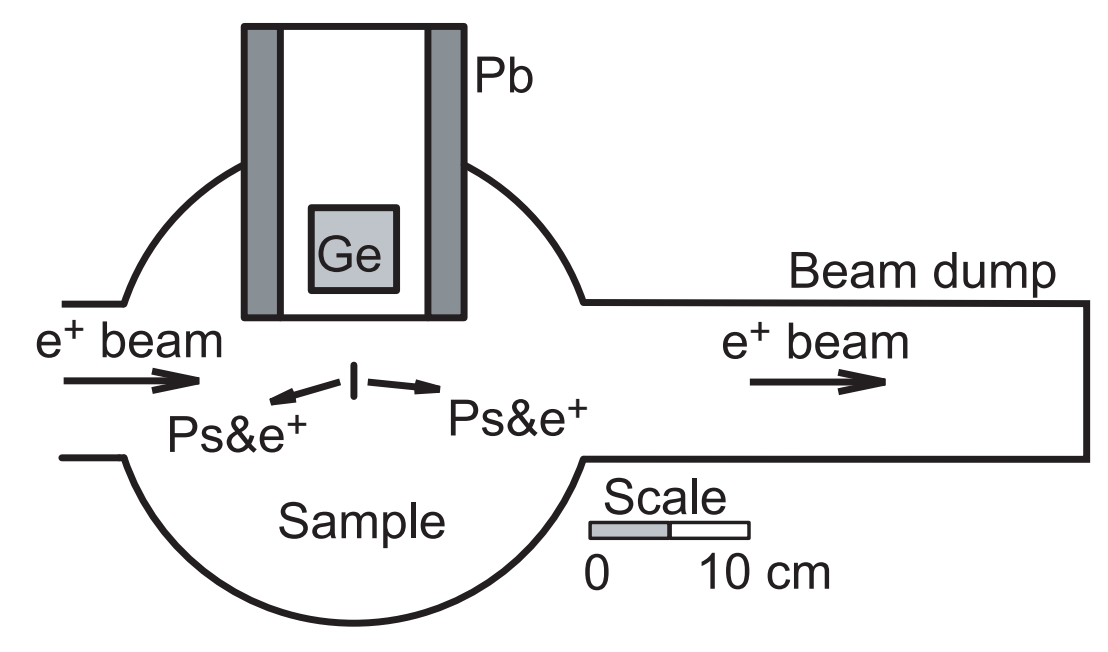


Figure 3.

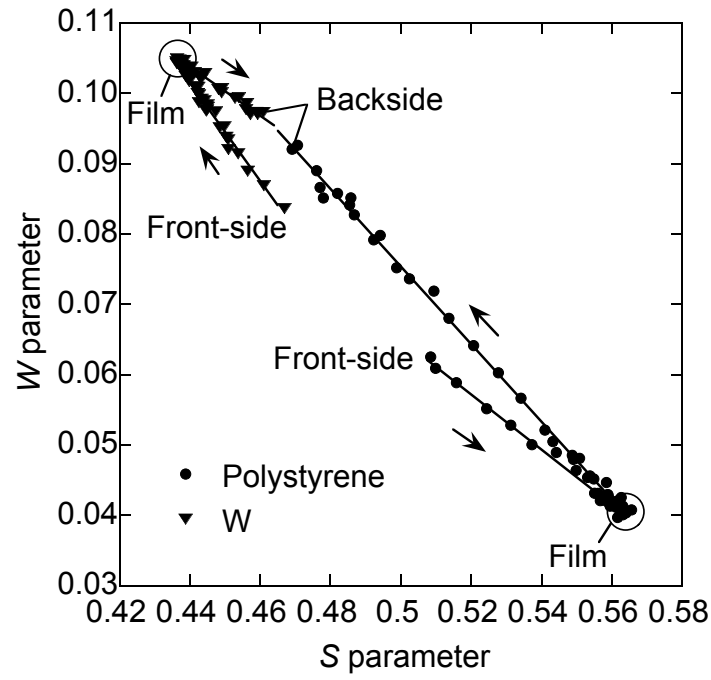

$S$ parameter 
Figure 4

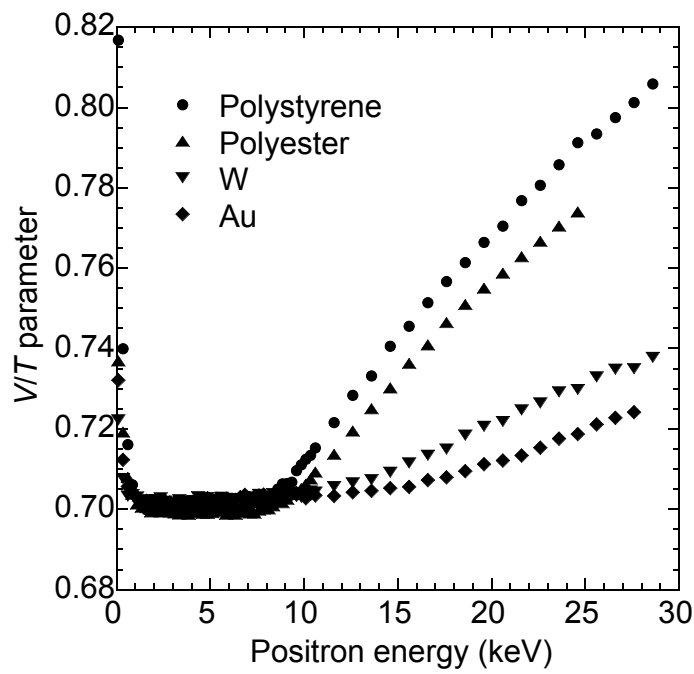

